\title{
Polarized EMC effect in the Thermodynamical Bag Model
}

\author{
K. Ganesamurthy* and R. Sambasivam ${ }^{\dagger}$ \\ Research Department of Physics, Urumu Dhanalakshmi College, Trichy - 620019, India.
}

(Received on 6 January, 2009)

\begin{abstract}
We determine the polarized quark distributions and structure functions for nuclear media such as $\mathrm{Li}^{7}$ and $\mathrm{Al}^{27}$ by using a phenomenological model known as Thermodynamical Bag Model. The evaluation of nuclear medium modifications to single nucleon structure function discusses the predictions of the polarized EMC effect. The deviation of polarized EMC from unpolarized case shows quenching of polarized quark distributions and proves the significance of this study as adding the spin observables will explore more about medium modification of nuclear structure and the nature of the strong interaction.
\end{abstract}

Keywords: EMC effect; Spin-dependence; structure functions

\section{INTRODUCTION}

Ever since the declaration of European Muon Collaboration (EMC) results [1] on the Deep Inelastic Scattering (DIS) of polarized leptons on polarized proton that the spin carried by the quarks is very small, considerable excitement started on what is known as 'EMC spin crisis'. The EMC proved undoubtedly that the quark distribution inside a nucleon differs significantly from that of the distribution inside a nucleus and the effect is known as EMC effect [2]. The Thermodynamical Bag Model (TBM) based evaluations [3, 4] yield the parton distribution functions with correct asymptotic behavior and adequately explains the small x-region where copious production of sea quarks and gluons arise as a natural consequence of this model. With the helicity of quarks, the model accounts for the spin puzzle of the nucleon caused by EMC and SMC [5] experiments. We are able to account for unpolarized EMC effect [6] by using TBM.

In this paper applying this framework we make predictions for polarized EMC effect. Initially the spin structure functions of proton and neutron are evaluated. In the convolution model, following the approach of Akulinichev et al. [7] the spin structure functions for nuclear media such as $\mathrm{Li}^{7}$ and $\mathrm{Al}^{27}$ are evaluated. The results of quark spin sums for a proton bound to the nuclear media are compared with that of the theoretical predictions using Nambu-Jona-Lasinio (NJL) model by Cloet et al. [8, 9].

\section{THEORETICAL ANALYSIS}

TBM as a modified form of MIT bag model [10] treats the quarks and gluons as fermions and bosons respectively. Considering the bag as an unpolarized proton, the four equations

\footnotetext{
*Electronic address: udckgmesify.com

†Electronic address: udcsamesify.com
}

of state are obtained in TBM as

$$
\begin{aligned}
{[\varepsilon(T) V+B V]^{2} } & =W^{2}=M^{2}+2 M v-Q^{2} \\
6\left(n_{u}-n_{\bar{u}}\right) & =\mu_{u} T^{2}+\frac{\mu_{u}^{3}}{\pi^{2}} \\
6\left(n_{d}-n_{\bar{d}}\right) & =\mu_{d} T^{2}+\frac{\mu_{d}^{3}}{\pi^{2}} \\
P & =\frac{\varepsilon(T)}{3}-B=0
\end{aligned}
$$

where $\varepsilon(T)$ is the energy density of the system at a temperature $T, V$ the volume of the bag, $B$ bag constant, $W$ the mass of the excited nucleon at temperature $T, v$ the energy transfer, $Q^{2}$ the square of four momentum transfer, $M$ the nucleon mass at $T=0,\left(n_{u}-n_{\bar{u}}\right)$ and $\left(n_{d}-n_{\bar{d}}\right)$ represent $u$ and $d$ valence quarks number density respectively, $\mu_{u}$ and $\mu_{d}$ are the chemical potentials of $u$ and $d$ quarks, and $P$ the pressure required to confine the partons inside the bag.

Treating the quarks as particles of zero rest mass, in the rest frame, the number of $u$-quarks with momentum lying between $p_{r}$ and $p_{r}+d p_{r}$ is given by

$$
n_{u}\left(p_{r}\right)=\frac{g V_{r}}{8 \pi^{3}} \frac{1}{\exp \left[\frac{1}{T}\left(\varepsilon_{r}-\mu_{u}\right)\right]+1}
$$

where $V_{r}$ denotes the nucleon volume, $\varepsilon_{r}$ the energy in rest frame, $\mu_{u}$ the chemical potential and $g$ the degeneracy factor all measured in the rest frame. Considering the nucleon to be moving with very high momentum along the $z$-axis, then in the moving frame or Infinite Momentum Frame (IMF) the Fermi distribution function of the $u$-quark can be written as

$$
n_{u}\left(p_{m}\right)=\frac{g V_{m}}{8 \pi^{3}} \frac{1}{\exp \left[\frac{1}{T_{m}}\left(\varepsilon_{m}-\mu_{u, m}\right)\right]+1}
$$

all quantities measured in moving frame denoted by the symbol $m$. The exponential factor is a dimensionless quantity and is invariant under Lorentz transformation and hence

$$
\frac{\varepsilon_{r}-\mu_{u}}{T}=\frac{\varepsilon_{m}-\mu_{u, m}}{T_{m}}
$$

Since the direction of motion of the nucleon is in the $z$-axis and the quarks are treated as particles with zero rest mass, the energy of the quark in the moving frame can be expressed in terms of its longitudinal and transverse momenta $p_{z}$ and $p_{t}$. 
On transforming the relation from lab frame to IMF we arrive at the relation

$$
\varepsilon_{m} \approx\left(p_{l}^{2}+p_{t}^{2}\right)^{1 / 2}
$$

Taking the longitudinal momentum of the quark as the fraction $x$ of the nucleon momentum, and Mac and Ugaz [11] for the transformation of volume $V_{r}$ in the rest frame to the volume $V_{m}$ in the moving frame and integrating over the transverse momentum in order to obtain the distribution function in terms of the longitudinal momentum, we finally get in the IMF the quark distribution as a function of chemical potential and temperature which can be written as

$$
u(x)=\frac{6 M^{2} V x T}{4 \pi^{2}} \ln \left[1+\exp \left\{\frac{1}{T}\left(\mu_{u}-\frac{M x}{2}\right)\right\}\right]
$$

Similarly the distribution functions for the $\bar{u}, d$ and $\bar{d}$ quarks are obtained as

$$
\begin{aligned}
& \bar{u}(x)=\frac{6 M^{2} V x T}{4 \pi^{2}} \ln \left[1+\exp \left\{\frac{1}{T}\left(-\mu_{u}-\frac{M x}{2}\right)\right\}\right] \\
& d(x)=\frac{6 M^{2} V x T}{4 \pi^{2}} \ln \left[1+\exp \left\{\frac{1}{T}\left(\mu_{d}-\frac{M x}{2}\right)\right\}\right] \\
& \bar{d}(x)=\frac{6 M^{2} V x T}{4 \pi^{2}} \ln \left[1+\exp \left\{\frac{1}{T}\left(-\mu_{d}-\frac{M x}{2}\right)\right\}\right]
\end{aligned}
$$

Here the distribution functions of antiquarks are obtained by reversing the sign of the chemical potential in the corresponding quark distribution functions. The equations (9)(12) involve the nucleon mass $M$. There is an intimate relation between $x$ and $T$ for a fixed $Q^{2}$. The smaller the value of $x$, the greater is the temperature $T$. According to Bjorken scaling, at lower values of $x$, energy transfer is very much greater than the momentum transfer. This represents the excited state of the target nucleon system. In our theoretical observations, the temperature of the bag model system starting approximately from $150 \mathrm{MeV}$ at lower values of $x$, decreases and tends to a minimum value as $x \rightarrow 1$. At finite $Q^{2}$, the invariant mass $W$ of the final hadronic state depends on $x$ and $Q^{2}$ that characterize the DIS.

The spin dependent structure functions of proton $g_{1}^{p}(x)$ and neutron $g_{1}^{n}(x)$ using the above four equations are given by

$$
\begin{aligned}
& g_{1}^{p}(x)=\frac{1}{2}\left[\left(\frac{4}{9} \Delta u(x)+\frac{1}{9} \Delta d(x)\right)\right] \\
& g_{1}^{n}(x)=\frac{1}{2}\left[\left(\frac{1}{9} \Delta u(x)+\frac{4}{9} \Delta d(x)\right)\right]
\end{aligned}
$$

where the spin distribution functions $\Delta u(x)$ and $\Delta d(x)$ are given by

$$
\begin{aligned}
& \Delta u(x)=\cos 2 \theta(x)\left[\{u(x)+\bar{u}(x)\}-\frac{2}{3}\{d(x)+\bar{d}(x)\}\right] \\
& \Delta d(x)=-\cos 2 \theta(x)[\{d(x)+\bar{d}(x)\} / 3]
\end{aligned}
$$

where

$$
\cos 2 \theta(x)=\left[1+\frac{H_{0}}{\sqrt{x}}(1-x)^{2}\right]^{-1}
$$

is known as the spin dilution factor. $H_{0}$ is a free parameter and is so chosen that Bjorken sum rule defined by

$$
\int_{0}^{1}\left(g_{1}^{p}-g_{1}^{n}\right) d x=\frac{1}{6} \frac{g_{A}}{g_{V}}
$$

is satisfied. Here $g_{A}$ and $g_{V}$ are the axial vector and vector coupling constants in neutron $\beta$ decay. It was already proved [3] that the number of quarks and anti-quarks of a particular flavor and helicity obtained by performing the integration in the rest frame or in the IMF is essentially the same.

\section{EVALUATION OF POLARIZED EMC EFFECT}

In this work, we concentrate on TBM predictions for spin dependent nuclear quark distributions and hence the polarized EMC effect. The EMC ratio is the nuclear spin structure function $g_{1}^{A}$ divided by the deuteron spin structure function $g_{1}^{d}$ where $g_{1}^{d} \approx g_{1}^{p}+g_{1}^{n}$. In the convolution model [7], the calculation of the nuclear spin structure function $g_{1}^{A}$ takes into account the influence of the nucleon binding energy and Fermi motion, and is provided by

$$
g_{1}^{A}(x)=\int f^{A}(z) g_{1}^{N}(x / z) d z
$$

where $f^{A}(z)$ describes the momentum and energy distribution of nucleons and $g_{1}^{N}$ is for single nucleon structure function. For the description of $g_{1}^{N}(x / z)$ in terms of quark degrees of freedom, the distance scale $x$ is modified to the rescaling variable $\eta=x / z$, which increases with mass number $A$. In order to calculate the effect of nuclear binding on the structure function, the momentum spectrum of the target nucleons has to be evaluated. In the simple Fermi gas model, the momentum distribution $\left(3 / 4 \pi k_{f}\right)$ is constant up to the maximum Fermi momentum $k_{f}$ and is zero above $k_{f}$. The momentum distribution inside the nucleus within the Fermi momentum can be written as

$$
f^{A}(z)=(3 / 4)\left(M / k_{f}\right)^{3}\left[\left(k_{f} / M\right)^{2}-(z-\eta)^{2}\right]
$$

for $-k_{f}<z<+k_{f}$ and $f^{A}(z)=0$ otherwise and this leads to the observed depletion at medium $\mathrm{x}$ as observed by the EMC effect. Using (19) and (20) the structure function ratio of nuclear media such as $\mathrm{Li}^{7}$ and $\mathrm{Al}^{27}$ to deuterium is obtained for $Q^{2}=5 G e V^{2}$ as

$$
\frac{g_{1}^{A}}{g_{1}^{d}}=\frac{\int f^{A}(z) g_{1}^{N}(x / z) d z}{g_{1}^{d}(x)}
$$

\section{RESULTS AND DISCUSSION}

Initially the polarized up and down quark distributions $\Delta u^{A}(x)$ and $\Delta d^{A}(x)$ in the nuclei such as $\mathrm{Li}^{7}$ and $\mathrm{Al}^{27}$ are evaluated using the convolution relation

$$
\Delta q^{A}(x)=\int f^{A}(z) \Delta q(x / z) d z
$$


Here the polarization of quarks is taken into account along with nuclear binding and Fermi motion. Unlike parameterized forms this model uses statistical distribution. Fig. 1 shows the light cone momentum fraction carried by the polarized quarks in the nuclear medium as expected. From Fig. 2 we find that the ratio of the polarized quark distributions in nuclear medium to the corresponding free polarized quark distribution $\left(\Delta u^{A} / \Delta u\right.$ and $\left.\Delta d^{A} / \Delta d\right)$ also show nuclear quenching effect on individual quark flavors and find that the effects are approximately equal over the valence quark region. The natures of the curves show resemblance with EMC effect. Fig. 3 and Fig. 4 provide polarized EMC effect in the studied nuclear media. In Fig. 4, SLAC-E139 [12] data for unpolarized structure function ratio averaged over $Q^{2}$ is shown for comparison. We observe that the ratio in the polarized case is less than the unpolarized case. Hence, the possible nuclear quenching effect on the polarized quarks is clearly established. However, the slight increase in the high fractional momentum carried by a free nucleon (as observed from Deuterium) at medium $x(0.3<x<0.7)$, show the occurrence of EMC effect.

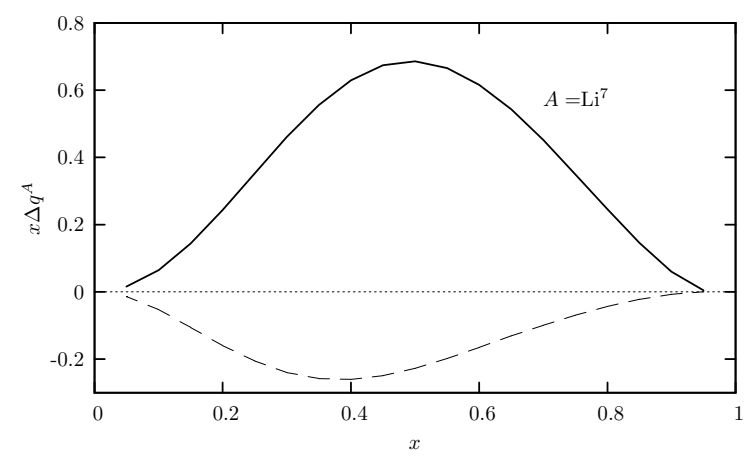

FIG. 1: Momentum distribution of polarized quarks in $\mathrm{Li}^{7}$. The solid line represents the distribution of polarized $u$-quarks and dashed line represents the distribution of polarized $d$-quarks.

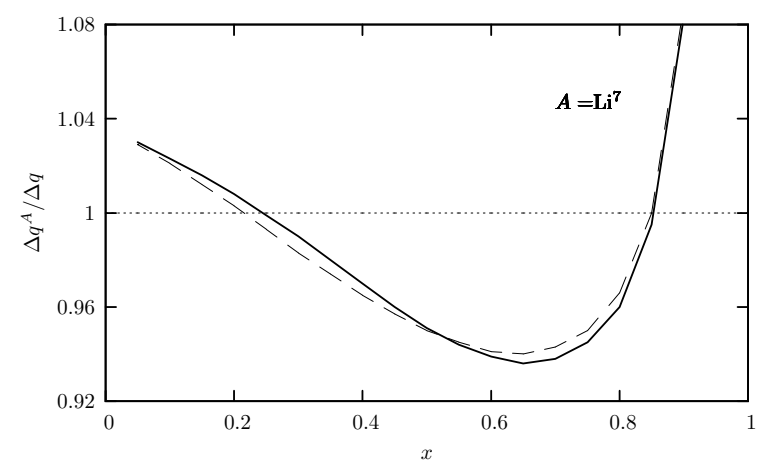

FIG. 2: EMC ratio of the polarized quark distribution in $\mathrm{Li}^{7}$. The solid line represents the ratio of polarized $u$-quark distribution in the nuclear medium $\mathrm{Li}^{7}$ to that of the free one. The dashed line stands for polarized $d$-quark.

As the mass number is increased, the difference between the two EMC ratios becomes more pronounced. At high values of $x(x>0.7)$ the dominance of Fermi motion almost

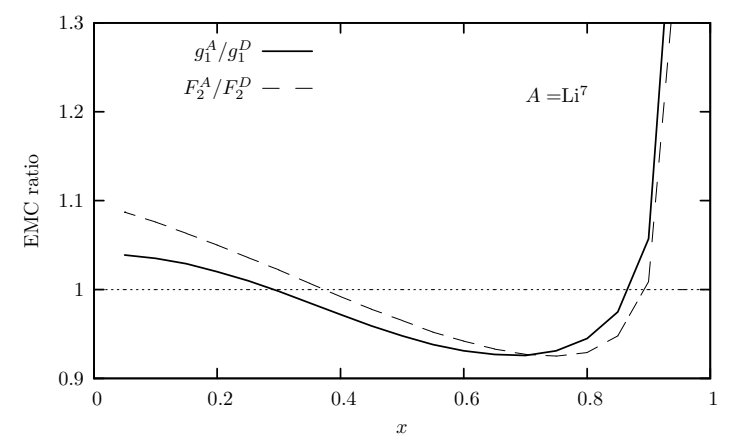

FIG. 3: Polarized and Unpolarized EMC effect in $\mathrm{Li}^{7}$. The solid line represents the EMC ratio of the nuclear spin structure function of $\mathrm{Li}^{7}$ to deuterium and the dashed line that of unpolarized structure functions ratio. Both are evaluated at $Q^{2}=5 \mathrm{GeV}^{2}$.

decreases the deviation and we do not find much difference. At very low values of $x$, the dominance of sea quarks is a natural consequence of this model. A physical measure of this graph, that shows low values, in medium values of $x$ may provide some insight into the polarized EMC case. Accordingly, Fig. 3 and Fig. 4 show the predicted polarized EMC effect using TBM. The spin sums of the bound nucleon are listed in the Table I and the results are compared with $[8,9]$. The nuclear spin sum represented by $\Sigma=\Delta u^{A}(x)+\Delta d^{A}(x)$ and axial coupling $g_{A}=\Delta u^{A}(x)-\Delta d^{A}(x)$ contain information on both nuclear and quark effects. The quantities are determined by performing the integration for the entire range of Bjorken variable. The values reasonably agree well. In evaluating the spin structure functions, our efforts to satisfy the Bjorken sum rule as defined by equation (18), lead our results of Table 1 to agree themselves within two or three significant digits. The decreasing values as mass number increases show the quark spin sums are quenched in the nuclear medium and the decrease of $g_{A}$ is also observed in nuclear $\beta$ decay studies.

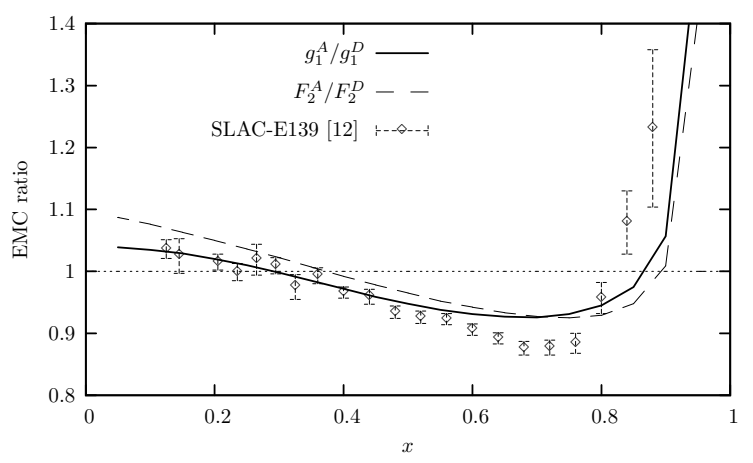

FIG. 4: Polarized EMC effect and unpolarized EMC effect for nucleus $\mathrm{Al}^{27}$ compared with experimental data SLAC-E139 [12]. 
TABLE I: Quark spin sums for a proton bound in the nuclear medium

\begin{tabular}{|l|c|c|c|c|c|c|c|c|}
\hline \multirow{2}{*}{$\begin{array}{c}\text { Nuclear } \\
\text { Medium }\end{array}$} & \multicolumn{2}{|c|}{$\Delta u^{A}(x)$} & \multicolumn{2}{c|}{$\Delta d^{A}(x)$} & \multicolumn{2}{c|}{$\Delta u^{A}(x)+\Delta d^{A}(x)$} & \multicolumn{2}{|c|}{$g_{A}$} \\
\cline { 2 - 9 } & TBM & Ref [8,9] & TBM & Ref [8,9] & TBM & Ref [8,9] & TBM & Ref [8,9] \\
\hline $\mathrm{Li}^{7}$ & 0.844 & 0.910 & -0.375 & -0.290 & 0.469 & 0.620 & 1.219 & 1.190 \\
\hline $\mathrm{Al}^{27}$ & 0.841 & 0.870 & -0.374 & -0.280 & 0.467 & 0.590 & 1.216 & 1.150 \\
\hline
\end{tabular}

\section{CONCLUSIONS}

In summary, TBM as a modified form of MIT bag model is used in initially establishing the momentum distribution of polarized quarks in nuclear media such as $\mathrm{Li}^{7}$ and $\mathrm{Al}^{27}$. These two nuclei are chosen as good choices with a single va- lence proton for polarized studies in earlier references. So, in our case, the polarized EMC effect measurements are taken into account. The quarks distribution and structure function show EMC effect and quenching of quark spin sums. Further studies may provide interesting path for nuclear strong interaction.
[1] J. Ashman et al. [E M Collaboration], Phys. Lett. B 206, 364 (1988), Nucl. Phys. B 328, 1 (1989).

[2] J. J. Aubert et al. [E M Collaboration], Phys. Lett. B 123, 275 (1983).

[3] V. Devanathan, S. Karthiyayini, and K. Ganesamurthy, Mod. Phys. Letts. A 9, 3455 (1994), M. Rajasekaran et al. Mod. Phys. Lett. A 5, 473 (1990).

[4] K. Ganesamurthy et al. Z. Phy. C 52, 589 (1991).

[5] B. Adeva et al. [S M Collaboration], Phys. Lett. B 302, 533 (1983), D. Adams et al. CERN-PPE/94-57, Phys. Lett. B (1994).

[6] K. Ganesamurthy, and R. Sambasivam, Turk. J. Phys. 32, 175
(2008).

[7] S. V. Akulinichev, S. Shlomo, S. A. Kulagin, and G. M.Vagradov, Phy. Rev. Lett. 55, 2239 (1985), Phys. Lett. B 158, 485 (1985)

[8] C. Cloet, W. Bentz, and A. W. Thomas, Phy. Lett. B 642, 210 (2006).

[9] W.Bentz et al. Progr. Part. Nuc. Phy., 61, 238 (2008), [arXiv:0711.0392v1(nucl-th)]

[10] A. Chodos et al. Phys. Rev. D9, 3471 (1974).

[11] E. Mac, and E.Ugaz, Z. Phy. C 43, 655 (1989).

[12] J. Gomez et al., Phy. Rev. D 49, 4348 (1994). 